\title{
Comparative genomics reveals a widespread distribution of an exopolysaccharide biosynthesis gene cluster among Vibrionaceae
}

\author{
Lou Lebellenger, Véronique Verrez-Bagnis, Delphine Passerini and Christine Delbarre-Ladrat * (1)
}

\begin{abstract}
Objectives: The eps locus in Vibrio diabolicus is involved in the production of the biotechnologically valuable HE800 EPS. In this study, the distribution and diversity of similar eps gene clusters across Vibrionaceae and its variability in relation to phylogenetic relationship were investigated. The aim was to provide a better knowledge of the eps gene cluster importance and to facilitate discovery of new EPS with potent interesting bioactivities.

Results: Seventy percent of the 103 genome sequences examined display such an eps locus with a high level of synteny. However, genetic divergence was found inside some monophyletic clades or even between some strains of the same species. It includes gene insertions, truncations, and deletions. Comparative analysis also reveals some variations in glycosyltransferase and export systems genes. Phylogenetic analysis of the Vibrionaceae eps gene clusters within Vibrionaceae suggests a vertical transfer by speciation but also pinpoints rearrangement events independent of the speciation.
\end{abstract}

Keywords: Vibrionaceae, Exopolysaccharide, Genetic biodiversity, Biosynthesis, Vibrio diabolicus, HE800 EPS

\section{Introduction}

The animal glycosaminoglycans are glycopolymers with key roles in cell physiology and pathologies [1]. The marine bacterium Vibrio diabolicus synthesizes the HE800 EPS, which shares some structure and bioactivity features with the glycosaminoglycan hyaluronan (HA) [2-4]. Thanks to its visco-elastic properties and biological properties on the cartilage and skin, HA is used in osteoarthritis treatment, ophthalmology, wound healing and in cosmetics. HE800 EPS has demonstrated its efficiency in bone and skin regeneration [5-7]. Its biosynthesis eps gene cluster has been identified (Fig. 1) [8] and appears as a good model to investigate eps loci in marine bacteria and find innovative glycosaminoglycan-mimetics for human health.

\footnotetext{
*Correspondence: cladrat@ifremer.fr

Ifremer, Atlantique Center, Biotechnology and Marine Resources Unit, Marine Ecosystems and Marine Molecules for the Biotechnologies Laboratory, Rue de l'lle d'Yeu, BP21105, 44311 Nantes Cedex 3, France
}

In this study, we investigated the distribution of orthologous eps gene clusters across Vibrionaceae and gene variations in relation to phylogenetic relationship.

\section{Main text \\ Methods \\ Assembly collection}

One hundred and three publicly available genome sequences of Vibrionaceae members, that cover several clades, were selected (Additional file 1).

Assemblies were obtained from NCBI [9] and three files were used: rna_from_genomic.fna for $16 \mathrm{rDNA}$ based phylogeny, protein.faa for homology search and cds_from_genomic.fna for genomic context evaluation.

\section{Identification of the eps orthologous clusters}

Proteins orthologous to $V$. diabolicus HE800 biosynthetic cluster were searched in the downloaded genomes by blastp comparisons using the standalone BLAST +2.2 .30$ 


\section{V. diabolicus eps cluster}

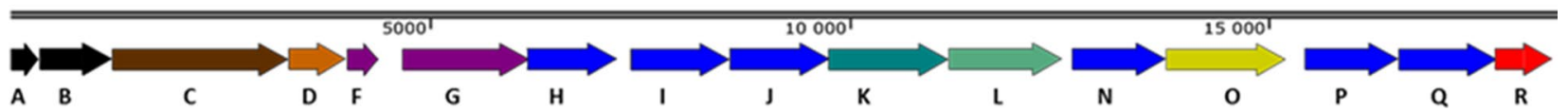

Fig. 1 Genomic structure of eps cluster of $V$. diabolicus. Scale: base pairs. Genes are labeled after the Aliivibrio fischeri orthologous genes [16].

Encoded proteins are: A: anti-sigma factor antagonist; B: regulatory protein; C: export protein (Wza); D: chain length regulator (WzC); F: sensor kinase; G: response regulator, transcriptional activator; H, I, J, N, P, and Q: glycosyltransferases; K: flippase (Wzx); L: polymerase (Wzy); O: chain length determinant (Wzz domain); R: undecaprenyl phosphate sugar phosphotransferase (priming GT)

package [10]. A local database gathering the 103 genomes was formatted with the makeblastdb program; a multifasta file containing the $16 \mathrm{~V}$. diabolicus eps genes was used as the blastp query.

Alignment length ratios were calculated as follows: MinLrap $=\mathrm{Lmatch} / \min (\operatorname{Lprot} 1$, Lprot 2$)$ and MaxLrap $=$ Lmatch $/ \max (\operatorname{Lprot} 1, \quad$ Lprot2 $)$ where Lmatch $=$ match length, Lprot1 (or Lprot2) $=$ protein 1 (or 2) length and $\min$ (or max) = minimum (or maximum) of the two values [11]. These values indicate, when both are equal to 1 , that both whole proteins align. Alignment length ratios (close to 1 ), similar protein sizes, low expectation value $\left(<10^{-25}\right)$ and identity percentage (above $40 \%)$ were taken into account to identify the first protein (usually A); genomic context was further inspected to evaluate if neighboring genes encode proteins which also share homology with $V$. diabolicus cluster.

\section{In silico analyses of the protein coding sequence sets}

Proteins encoded by the genes inserted between D and F were identified by Blast search against the NCBI database [12]. A phylogenetic tree was constructed using a concatenate of proteins; sequences were aligned with COBALT (https://www.ncbi.nlm.nih.gov/tools/cobalt/) and phylogenetic analyses were conducted with MEGA software v7 using the Neighbor-Joining method [13, 14].

\section{Multilocus sequence analysis (MLSA)}

Five housekeeping genes were used for strains MLSA phylogeny [15] (Additional file 2). Genes were aligned with MAFFT version 7 . Because not all gene sequences are full length, positions 1-562, 227-893, 391-892, 4421027, and 448-1073 ( $V$. diabolicus numbering) of $p y r H$, gapA, mreB, gyrB, and topA genes, respectively, were concatenated. Phylogenetic tree was constructed with MEGA 7 using the Neighbor-Joining method [13, 14].

\section{Results and discussion}

\section{Distribution of the eps gene cluster in Vibrionaceae}

Seventy-two orthologous eps clusters were discovered, while none could be found in the remaining 31 genomes, which include Grimontia and Salinivibrio members (Fig. 2a, Additional files 3, 4). V. ichthyoenteri was found to possess an eps gene cluster but it was not further analyzed because the cluster is split at the ends of two distinct contigs.

\section{Variability of the eps gene cluster}

The organization of the gene clusters was highly conserved, particularly in two syntenic blocks: the $5^{\prime}$ end of the cluster (A, B and C genes) and the $3^{\prime}$ end which always encompasses gene $\mathrm{R}$. The species possessing the eps cluster the most similar to the $V$. diabolicus one, are $V$. antiquarius Ex25, V.alginolyticus and $V$. parahaemolyticus (Fig. 2a). The most conserved proteins are A, B, $\mathrm{C}$ and $\mathrm{G}$, which are involved in regulation, $\mathrm{O}$ which is responsible for chain length regulation and $\mathrm{R}$, which initiates polymerization; they shared more than $70 \%$ identity with $V$. diabolicus orthologs (Fig. 2b). The A protein is an anti-sigma factor antagonist and a key regulator of biofilm formation; the deletion of A gene in $V$. fischeri $(\operatorname{syp} A)$ inhibits biofilm formation and thus squid colonization [16]. The good conservation of both $B$ and $C$ proteins suggests they are important for the EPS biosynthesis, although their functions remain undetermined. D protein which functions as a chain length regulator [17] is conserved; an identity of about $50 \%$ with that encoded by $V$. diabolicus eps cluster suggests that its activity can be variable (Fig. 2b, Additional file 3).

The $\mathrm{G}$ gene, that codes for a 654 -dependent activator of syp locus transcription in A. fischeri, is also conserved [18-22] (Fig. 2b, Additional file 3). F, the other regulatory protein, is a hybrid sensor kinase which phosphorylates SypE and SypG in order to regulate biofilm formation in $V$. fischeri $[20,23]$. F protein seems to be particularly subjected to variation (Fig. 2b), as it appears truncated in $V$. diabolicus eps cluster, whereas some other species carry a full copy $[8,17]$.

The $V$. diabolicus eps cluster encompasses 6 glycosyltransferases (GT), which biosynthesize the EPS repeating unit, and one priming GT (R), which initiates the EPS biosynthesis. Among the considered Vibrionaceae 


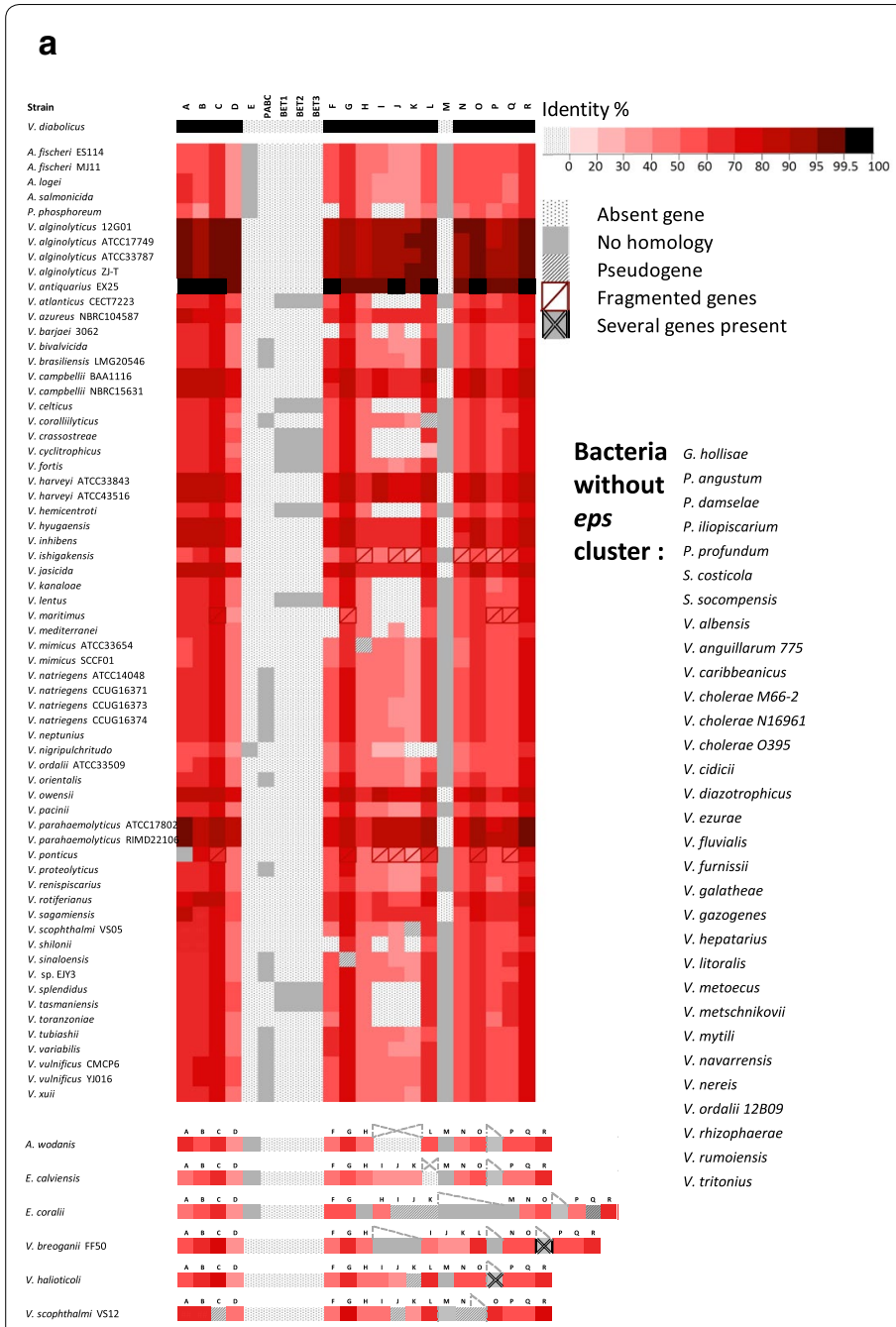

b
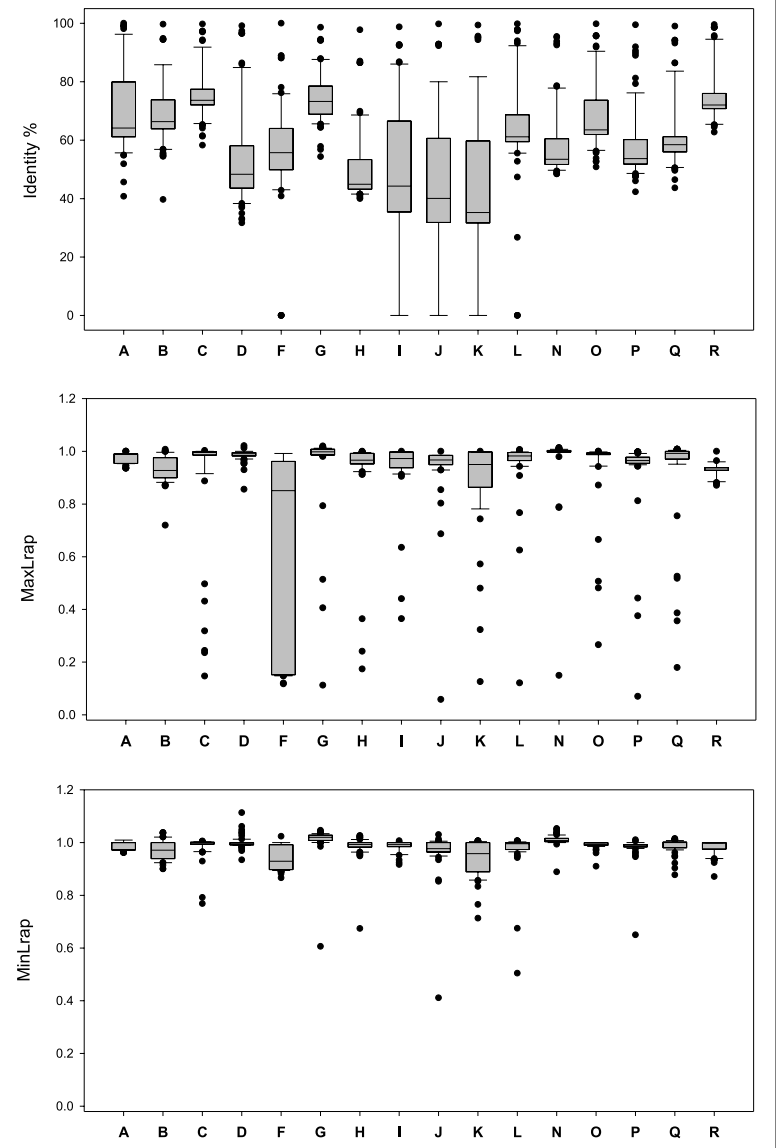

Fig. 2 Biodiversity of the eps cluster among Vibrionaceae. a Protein components of the orthologous clusters with the identity \% to V. diabolicus protein. Color legend is located at the top right portion, red level shows increasing identity by from light red to black. Dotted position are absent proteins, grey ones are proteins that do not exist in the HE800 cluster. b Boxplots showing distribution of data set from identity \%, MaxLrap and MinLrap of $A, B, C, D, F, G, H, I, J, K, L, N, O, P, Q$ and R proteins in comparison with V. diabolicus proteins. In the boxes, the 25th, 50th and 75th percentiles are indicated by the bottom, middle and top lines respectively. Whiskers show the 10th and 90th percentiles. Individual dots are the outliers. Absent genes were withdrawn from the min and maxLrap diagrams

strains, 16 are lacking at least one of the $V$. diabolicus GTs. The most often observed difference is the absence of both I and J in 12 eps clusters (Fig. 2a). I protein has only a weak role in the EPS production, adding a branch on the polysaccharide backbone late in the biosynthesis process [17]. In addition, several GT genes are fragmented or annotated as pseudogenes. In $V$. breoganii FF50 and $V$. halioticoli, the P, Q and R gene group is separated from the rest of the eps cluster by, respectively, a $60 \mathrm{~kb}$-long and a $5 \mathrm{~kb}$-long sequence. Therefore, these genes may not be transcribed together with other eps genes. However, the PQR segment might be sufficient to synthesize some oligosaccharides, as in
A. fischeri, the syp $P Q R$ segment constitutes an operon [24]. When present, H, I and J proteins display about $50 \%$ identity with the corresponding $V$. diabolicus ones. $\mathrm{N}, \mathrm{P}$ and $\mathrm{Q}$ are slightly more conserved (about $60 \%$ identity) suggesting only slight putative differences in substrate and acceptor specificities. P, Q and $\mathrm{R}$ proteins exhibit an overall very high conservation across Vibrionaceae. They have been hypothesized to be related to the repeating unit biosynthesis [8], which could thus likely be predicted to be rather similar in Vibrionaceae EPS.

The HE800 EPS export system involves the periplasmic protein Wza (C protein), the oligosaccharide translocase 
Wzx (K protein), the polymerase Wzy (L protein) and the putative polysaccharide biosynthesis chain length regulator Wzc (O protein). It has been previously established that $\mathrm{K}$ protein is essential for EPS repeating unit translocation across the inner membrane $[8,17]$. But 15 of the Vibrionaceae studied species are devoid of this protein, suggesting that they are not able to produce, or at least export, an EPS molecule [17]. L gene is absent in Enterovibrio calviensis, and classified as pseudogene in $V$. coralliilyticus. Both genes $\mathrm{K}$ and $\mathrm{L}$ are absent in $V$. nigripulchritudo (Fig. 2a). In all these strains, if the biosynthesis of the repeating unit occurs, it may accumulate most likely in the cytosol. Indeed, the proteins encoded by their eps cluster do not enable the repeating unit to be translocated across the membrane nor polymerized $[8$, 25].

Between $\mathrm{D}$ and $\mathrm{F}$ genes, additional individual genes or a group of three genes were found in several of the studied Vibrionaceae (Fig. 2a). One of these proteins (E) is homologous to A. fischeri SypE which is a two-component response regulator protein inhibiting SypG-mediated phenotypes and biofilm formation [19]. Another individual gene codes for a periplasmic component of an $A B C$ type phosphate/phosphonate transport system (PABC). Phosphonates can be found as side groups on several macromolecules including polysaccharides [26]. On the other hand, EPS are known to form a slime around cells to sequester several compounds and could therefore be involved in bioremediation. But it is not clear why only the PABC periplasmic component is present while some Gram-negative bacteria possess a full copy of the phosphonate biosynthetic operon beside EPS biosynthesis genes [26]. For eight of the strains studied, three genes, coding for a glycine betaine/L-proline ABC transporter substrate-binding protein, a permease and an ATP-binding protein, are located between the genes D and F. As these proteins are homologous to the ProVWX components of the ProU transporter in Escherichia coli K12 [27], they may contribute to the uptake of glycine betaine which participates in bacterial osmoregulation, cryoprotection and protection against desiccation [28]. Betaine containing molecules can also constitute a source of phosphorus [29]. Several polysaccharides, especially the anionic ones, have been described to interact with glycine betaine compounds [30]. The presence of such transporter within EPS biosynthetic clusters could suggest adaptation of the bacterial strain to specific environmental conditions.

Enterovibrio coralii carries a glutamine-fructose6-phosphate aminotransferase between $\mathrm{H}$ and $\mathrm{N}$ genes. It catalyzes the rate-limiting step in the synthesis of UDP$\mathrm{N}$-acetylglucosamine [31,32] which is a precursor for both polysaccharide synthesis and cell growth in E. coli [33].

Several transposase and integrase genes have been identified in V. breoganii and in V. scophthalmi VS-12. These proteins allow insertion of mobile elements and thus recombination events [34, 35].

The syp locus of $A$. fischeri encodes the additional SypM, an $O$-acetyltransferase [17]. It has also been identified in 52 strains over the 103 studied ones (M, Fig. 2a). This could suggest the presence of $O$-acetyl groups in the final putative molecule.

\section{Phylogenetic relationships}

The MLSA phylogenetic tree (Fig. 3b) shows congruence in the clustering of the large majority of strains with trees previously described [15]. Concatenated proteins are generally clustered in the monophyletic clades (Fig. 3a). However some exceptions are noticed. $V$. natriegens species and $V$. sp. EJY3 are the sole strains of the Harveyi clade which possess the PABC protein. PABC protein was found in 17 strains belonging to 6 different clades (Harveyi, Orientalis, Nereis, Mediterranei, Coralliilyticus and Vulnificus); these clades also encompass other members possessing eps cluster that does not encode this protein. V. mytili is the only Harveyi clade member (over 25 ) that does not share the eps cluster. The concatenated proteins of $V$. nigripulchritudo (Nigripulchritudo clade) appear isolated as they miss $\mathrm{K}$ and $\mathrm{L}$ proteins. V. nigripulchritudo is also the sole Vibrio to have E protein which is also found in Fischeri, Enterovibrio and Phosphoreum species. $V$. vulnificus and $V$. mimicus are the only representatives of the Vulnificus and Cholerae clades, respectively, sharing the eps cluster. Nevertheless, $V$. mimicus seems to be an atypical species of Cholerae clade [36, 37]. All the 11 Splendidus clade species possess the eps cluster. Moreover, all studied species of seven clades examined (Coralliilyticus, Enterovibrio, Fischeri, Mediterranei, Nigripulchritudo, Scophthalmi, Splendidus) share the eps cluster. On the other hand, betaine $\mathrm{ABC}$ transporter genes were found only in the Splendidus clade with the exception of $V$. kanaloae and $V$. toranzoniae.

Blast search on the NCBI genome sequence database [12] excluding all Vibrionaceae species was used to infer the overall occurrence of the eps cluster. Only a few genomes contained homologs to the eps genes (Additional file 5). However, in these strains, the gene order is different, some gene blocks are inverted and differentially located on $(+)$ and $(-)$ DNA strands. In addition, several deletions/insertions are observed. The eps cluster examined in this paper thus appears as specific to Vibrionaceae and has likely been acquired by horizontal gene transfer in the few other bacteria sharing it. 


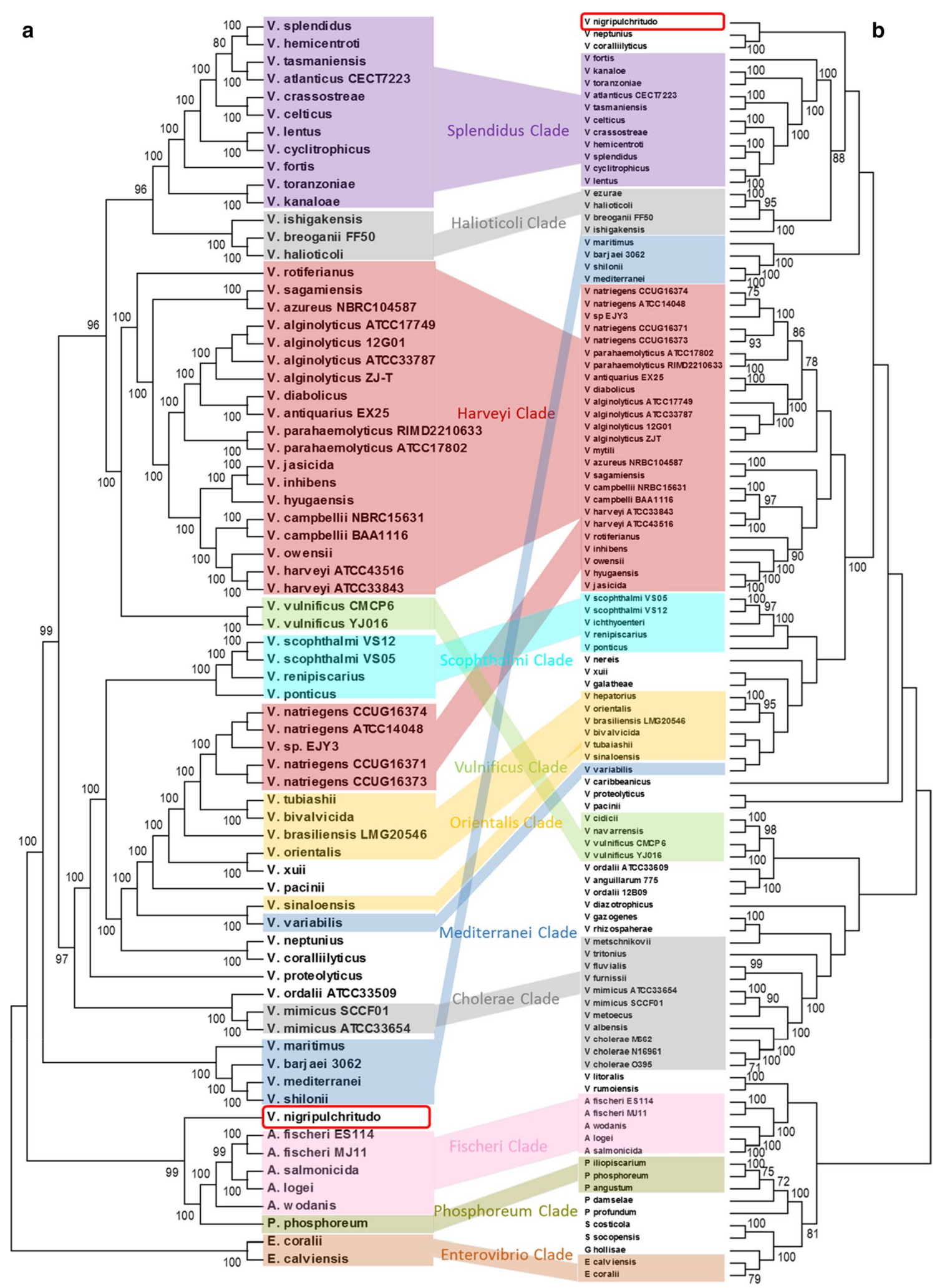

Fig. 3 Evolutionary relationships of concatenated proteins encoded by eps gene clusters (a) and MLSA phylogenetic relationships between strains (b). Main monophyletic clades are also indicated in both trees. Concatenated proteins were aligned with COBALT and MLSA five-gene dataset was aligned with MAFFT. Phylogenetic trees were constructed with MEGA version7 using the Neighbor-Joining method [13, 14] with the Poisson correction method for proteins [38] and the Jukes Cantor substitution model for MLSA [39]. Bootstrap values (1000 replicates) are shown next to the branches when higher than 70 [40] 


\section{Limitations}

This identification of a large number of orthologous eps clusters within the Vibrionaceae is interesting to obtain EPS glycosaminoglycan-like molecules with distinct structural features. But it necessitates further studies by isolating and characterizing the putative EPS produced to gain insight into the EPS structural features. This is a challenge because EPS production conditions and regulation mechanisms are not fully understood. The characterization of a large number of EPS molecules together with the biosynthesis gene cluster structure would provide a relevant basis to identify the genetic mechanisms of the biosynthesis and open the field of synthetic biology to produce glycosaminoglycan-mimetics.

\section{Additional files}

Additional file 1. List of strains studied. The assembly reference is indicated as well as clades.

Additional file 2. List of strains and sequence accession numbers used for the MLSA (multilocus sequence analysis).

Additional file 3. Proteins encoded by eps orthologous clusters in Vibrionaceae. Lines in green (E. coralii, V. breoganii FF50 and V. ichthyoenteri): clusters with specific genes as indicated, Lines in red ( $V$. ishigakensis, $V$. maritimus, V. ponticus): strains with replicated genes. Locus tags annotated as pseudogene are also identified.

Additional file 4. Biodiversity of the eps cluster among Vibrionaceae.

Additional file 5. Gene clusters retrieved from the NCBI genome database excluding Vibrionaceae that show homology to eps cluster.

\section{Abbreviations}

EPS: exopolysaccharide; HA: hyaluronan; MLSA: multilocus sequence analysis; PABC: $A B C$ type phosphate/phosphonate transport system.

\section{Authors' contributions}

$L L$ and DP conceived and carried out the analysis; assembly downloads and BlastP searches were performed by $C D L ; L L, C D L$ and VVB wrote the original manuscript. CDL coordinated the study. All the authors have made substantial, direct and intellectual contribution to the work. All authors read and approved the final manuscript.

\section{Acknowledgements}

We acknowledge M. Dols-Lafargue and A. Zykwinska who kindly accepted to read the final manuscript.

\section{Competing interests}

The authors declare that they have no competing interests.

\section{Availability of data and materials}

The data supporting this study are available on NCBI database and are included within the article and the additional files.

\section{Consent for publication}

Not applicable.

\section{Ethics approval and consent to participate} Not applicable.

\section{Funding}

This work is part of L. Lebellenger thesis supported with a fellowship from Ifremer and Région Pays de la Loire within the research network GlycoOuest.

\section{Publisher's Note}

Springer Nature remains neutral with regard to jurisdictional claims in published maps and institutional affiliations.

Received: 25 July 2017 Accepted: 31 January 2018

Published online: 06 February 2018

\section{References}

1. Lima M, Rudd T, Yates E. New applications of heparin and other glycosaminoglycans. Molecules. 2017;22(5):749.

2. Raguenes G, Christen R, Guezennec J, Pignet P, Barbier G. Vibrio diabolicus sp. nov., a new polysaccharide-secreting organism isolated from a deepsea hydrothermal vent polychaete annelid, Alvinella pompejana. Int J Syst Evol Microbiol. 1997;47(4):989-95.

3. Rougeaux H, Kervarec N, Pichon R, Guezennec J. Structure of the exopolysaccharide of Vibrio diabolicus isolated from a deep-sea hydrothermal vent. Carbohydr Resh. 1999;322(1-2):40-5.

4. Delbarre-Ladrat C, Sinquin C, Lebellenger L, Zykwinska A, Colliec-Jouault S. Exopolysaccharides produced by marine bacteria and their applications as glycosaminoglycan-like molecules. Front Chem. 2014;2:85.

5. Zanchetta P, Lagarde N, Guezennec J. A new bone-healing material: a hyaluronic acid-like bacterial exopolysaccharide. Calcif Tissue Int. 2003;72(1):74-9.

6. Senni K, Gueniche F, Changotade S, Septier D, Sinquin C, Ratiskol J, Lutomski D, Godeau G, Guezennec J, Colliec-Jouault S. Unusual glycosaminoglycans from a deep sea hydrothermal bacterium improve fibrillar collagen structuring and fibroblast activities in engineered connective tissues. Mar Drugs. 2013;11(4):1351-69.

7. Rederstorff E, Weiss P, Sourice S, Pilet P, Xie F, Sinquin C, Colliec-Jouault S, Guicheux J, Laïb S. An in vitro study of two GAG-like marine polysaccharides incorporated into injectable hydrogels for bone and cartilage tissue engineering. Acta Biomater. 2011;7(5):2119-30.

8. Goudenège $D$, Boursicot V, Versigny $T$, Bonnetot $S$, Ratiskol J, Sinquin C, LaPointe G, Le Roux F, Delbarre-Ladrat C. Genome sequence of Vibrio diabolicus and identification of the exopolysaccharide HE800 biosynthesis locus. Appl Microbiol Biotechnol. 2014;98(24):10165-76.

9. NCBI. https://www.ncbi.nlm.nih.gov/. Accessed Dec 2016.

10. Camacho C, Coulouris G, Avagyan V, Ma N, Papadopoulos J, Bealer K, Madden TL. BLAST+: architecture and applications. BMC Bioinform. 2009;10(1):421.

11. Microscope. https://microscope.readthedocs.io/en/latest/. Accessed 18 July 2017.

12. Blast. https://blast.ncbi.nlm.nih.gov/. Accessed Apr 2017.

13. Kumar S, Stecher G, Tamura K. MEGA7: molecular evolutionary genetics analysis version 7.0 for bigger datasets. Mol Biol Evol. 2016;33(7):1870-4.

14. Saitou N, Nei M. The neighbor-joining method: a new method for reconstructing phylogenetic trees. Mol Biol Evol. 1987;4(4):406-25.

15. Sawabe T, Ogura Y, Matsumura Y, Gao F, Amin AR, Mino S, Nakagawa S, Sawabe T, Kumar R, Fukui Y, et al. Updating the Vibrio clades defined by multilocus sequence phylogeny: proposal of eight new clades, and the description of Vibrio tritonius sp. nov. Front Microbiol. 2013;4:414.

16. Morris AR, Darnell CL, Visick KL. Inactivation of a novel response regulator is necessary for biofilm formation and host colonization by Vibrio fischeri. Mol Microbiol. 2011;82(1):114-30.

17. Shibata S, Yip ES, Quirke KP, Ondrey JM, Visick KL. Roles of the structural symbiosis polysaccharide (syp) genes in host colonization, biofilm formation, and polysaccharide biosynthesis in Vibrio fischeri. J Bacteriol. 2012;194(24):6736-47.

18. Yip ES, Geszvain K, DeLoney-Marino CR, Visick KL. The symbiosis regulator RscS controls the syp gene locus, biofilm formation and symbiotic aggregation by Vibrio fischeri. Mol Microbiol. 2006;62(6):1586-600.

19. Hussa EA, Darnell CL, Visick KL. RscS functions upstream of SypG to control the syp locus and biofilm formation in Vibrio fischeri. J Bacteriol. 2008;190(13):4576-83.

20. Yip ES, Grublesky BT, Hussa EA, Visick KL. A novel, conserved cluster of genes promotes symbiotic colonization and o54-dependent biofilm formation by Vibrio fischeri. Mol Microbiol. 2005;57(5):1485-98. 
21. Morris AR, Visick KL. Inhibition of SypG-induced biofilms and host colonization by the negative regulator SypE in Vibrio fischeri. PLoS ONE. 2013;8(3):e60076.

22. Morris AR, Visick KL. The response regulator SypE controls biofilm formation and colonization through phosphorylation of the syp-encoded regulator SypA in Vibrio fischeri. Mol Microbiol. 2013;87(3):509-25.

23. Norsworthy AN, Visick KL. Signaling between two interacting sensor kinases promotes biofilms and colonization by a bacterial symbiont. Mol Microbiol. 2015;96(2):233-48.

24. Ray VA, Eddy JL, Hussa EA, Misale M, Visick KL. The syp enhancer sequence plays a key role in transcriptional activation by the o54-fependent response regulator SypG and in biofilm formation and host colonization by Vibrio fischeri. J Bacteriol. 2013;195(23):5402-12.

25. Whitfield C. Biosynthesis and assembly of capsular polysaccharides in Escherichia coli. Annu Rev Biochem. 2006:75:39-68.

26. Villarreal-Chiu J, Quinn J, McGrath J. The genes and enzymes of phosphonate metabolism by bacteria, and their distribution in the marine environment. Front Microbiol. 2012;3:19.

27. Gul N, Poolman B. Functional reconstitution and osmoregulatory properties of the ProU ABC transporter from Escherichia coli. Mol Membr Biol. 2013;30(2):138-48.

28. Kapfhammer D, Karatan E, Pflughoeft KJ, Watnick PI. Role for glycine betaine transport in Vibrio cholerae osmoadaptation and biofilm formation within microbial communities. Appl Environ Microbiol. 2005;71(7):3840-7.

29. Park Yl, Buszko ML, Gander JE. Utilization of phosphocholine from extracellular complex polysaccharide as a source of cytoplasmic choline derivatives in Penicillium fellutanum. J Bacteriol. 1997;179(4):1186-92.

30. Covis R, Vives T, Gaillard C, Benoit M, Benvegnu T. Interactions and hybrid complex formation of anionic algal polysaccharides with a cationic glycine betaine-derived surfactant. Carbohydr Polym. 2015;121:436-48.
31. Badet B, Vermoote P, Le Goffic F. Glucosamine synthetase from Escherichia coli: kinetic mechanism and inhibition by N3-fumaroyl-L-2,3-diaminopropionic derivatives. Biochemistry. 1988;27(7):2282-7.

32. Milewski S. Glucosamine-6-phosphate synthase-the multi-facets enzyme. Biochim Biophys Acta. 2002;1597(2):173-92.

33. Yu H, Stephanopoulos G. Metabolic engineering of Escherichia coli for biosynthesis of hyaluronic acid. Metab Eng. 2008;10(1):24-32.

34. Polard $\mathrm{P}$, Chandler M. Bacterial transposases and retroviral integrases. Mol Microbiol. 1995;15(1):13-23.

35. Darmon E, Leach DRF. Bacterial genome instability. Microbiol Mol Biol Rev. 2014:78(1):1-39.

36. Davis BR, Fanning GR, Madden JM, Steigerwalt AG, Bradford HB, Smith $\mathrm{HL}$, Brenner DJ. Characterization of biochemically atypical Vibrio cholerae strains and designation of a new pathogenic species, Vibrio mimicus. J Clin Microbiol. 1981;14(6):631-9.

37. Gomez-Gil B, Thompson CC, Matsumura Y, Sawabe T, lida T, Christen R, Thompson F, Sawabe T. The family Vibrionaceae. In: Rosenberg E, DeLong EF, Lory S, Stackebrandt E, Thompson F, editors. The prokaryotes-gammaproteobacteria. 4th ed. Berlin: Springer; 2014. p. 659-747.

38. Zuckerkandl E, Pauling L. Evolutionary divergence and convergence in proteins. In: Bryson V, Vogel HJ, editors. Evolving genes and proteins. New York: Academic Press; 1965. p. 97-166.

39. Jukes TH, Cantor CR. Evolution of protein molecules. In: Munro HN, editor. Mammalian protein metabolism. New York: Academic Press; 1969. p. $21-132$.

40. Felsenstein J. Confidence limits on phylogenies: an approach using the bootstrap. Evolution. 1985;39(4):783-91.

\section{Submit your next manuscript to BioMed Central and we will help you at every step:}

- We accept pre-submission inquiries

- Our selector tool helps you to find the most relevant journal

- We provide round the clock customer support

- Convenient online submission

- Thorough peer review

- Inclusion in PubMed and all major indexing services

- Maximum visibility for your research

Submit your manuscript at www.biomedcentral.com/submit
() Biomed Central 